Delft University of Technology

\title{
Quality-aware control for optimizing meat supply chains
}

Sprong, J. P.; Lin, X.; Maestre, J. M.; Negenborn, R. R.

DOI

10.23919/ECC.2019.8795777

Publication date

2019

Document Version

Accepted author manuscript

Published in

Proceedings of the 18th European Control Conference (ECC 2019)

\section{Citation (APA)}

Sprong, J. P., Lin, X., Maestre, J. M., \& Negenborn, R. R. (2019). Quality-aware control for optimizing meat supply chains. In Proceedings of the 18th European Control Conference (ECC 2019) (pp. 3867-3872). IEEE . https://doi.org/10.23919/ECC.2019.8795777

\section{Important note}

To cite this publication, please use the final published version (if applicable).

Please check the document version above.

\section{Copyright}

Other than for strictly personal use, it is not permitted to download, forward or distribute the text or part of it, without the consent of the author(s) and/or copyright holder(s), unless the work is under an open content license such as Creative Commons.

\section{Takedown policy}

Please contact us and provide details if you believe this document breaches copyrights.

We will remove access to the work immediately and investigate your claim. 


\title{
Quality-Aware Control for Optimizing Meat Supply Chains
}

\author{
J.P. Sprong ${ }^{1}$, X. Lin ${ }^{1}$, J.M. Maestre ${ }^{2}$, R.R. Negenborn ${ }^{1}$
}

\begin{abstract}
Controlling food quality and reducing waste is one of the most challenging tasks in the food industry, as it is facing high rates of wastage, leading to negative environmental impact. This research focuses on improving the scheduling and control of the supply chain of Irish lamb meat using real-time quality and temperature information. Temperature controlled reefers and sensor technologies can be used to monitor and set the temperature during transport and storage. In order to minimize waste, while at the same time optimizing cooling and transport costs, a mathematical model is proposed. The model consists of two aspects. The quality aspect considers the shelf life of Irish lamb which is related to temperature. The logistic aspect considers supply chain scheduling. With this model, a strategy is proposed to determine the movements of meat, as well as the temperature setting of cooling equipment. Results of simulation experiments indicate a sustainable approach can reduce or even eliminate waste and decrease operational costs when real-time monitoring and control is used.
\end{abstract}

Keywords - Fresh food logistics, meat supply chain, shelf life, cooling costs, quality-aware modeling, mixed-integer linear programming.

\section{INTRODUCTION}

The United Nations estimates that annually $1 / 3$ of all food produced for human consumption is wasted. Costs of this food waste in the European Union alone are estimated at around 143 billion euros [1]. Food waste, food safety, and food quality are intimately linked with refrigeration: failing to keep perishable food in the desired temperature range can render the product inedible. Perishable foods include fresh fruits, vegetables, dairy, fish products, and meats. Focusing on the latter, meat production and consumption has grown a 4-5 fold since 1961 [2], while about $20 \%$ of all meat is wasted as estimated by the United Nations. If one now considers that for every kilogram of meat up to 130 times more water as compared to a kilogram of potatoes is needed and up to 9 times more $\mathrm{CO}_{2}$ is produced, the impact of this waste becomes more evident. This does not even consider the food consumption of livestock.

Meat is a perishable product with a short shelf life and therefore short selling times. Shelf life is dependent on the quality of meat, which is highly influenced by temperature [3], [4]. Hence, logistic decisions in the cold supply chain of meat are put under additional pressure. Information about

*Financial support by the H2020 ADG-ERC project OCONTSOLAR (ID 789051 ) and by the MINECO-Spain project DPI2017-86918-R is gratefully acknowledged.

${ }^{1}$ Department of Maritime and Transport Technology, Delft University of Technology, Delft, The Netherlands

2 Dept. Ingeniería de Sistemas y Automática, Universidad de Sevilla, Sevilla, Spain

Corresponding author: X. Lin x.lin@tudelft.nl temperature can be made available in real-time by sensors and communication technologies as shown by [4] and [5]. This information is beneficial when considered in decision making of logistics activities in supply chain management. Therefore, the objective of this paper is to develop a strategy to optimize meat supply chains with the consideration of cooling temperature and meat quality.

The paper is organized as follows. Section II presents a literature review on the quality and logistics of fresh food supply chains in general, and works on meat supply chains in particular. In Section III important factors in the degradation of the quality of meat are presented including a mathematical shelf life model. Additionally, the logistic aspects of the supply chain of meat are inspected and relevant stages are distinguished. Also, a mathematical model that considers both logistics and quality will be presented. Section IV presents an optimal control strategy to find a solution for the transport of meat through the supply chain considering logistics as well as quality. This strategy will be compared with the contemporary supply chain control. Finally, in Section V the research is concluded and directions for further research are provided.

\section{LITERATURE REVIEW}

Shelf life of meat is highly dependent on temperature. Temperature abuses in the cold supply chain of meat lead to an increase in growth and survival of bacteria, and thus lead to a decrease of quality and shelf life (see e.g., [4], [6], [7], [8]). Optimization of logistic processes considering transport and inventory in supply chains have been widely researched. Optimization of the meat supply chain, however, has received very little attention in literature. A multi-objective linear programming model for a generic beef logistics network problem is used in [9]. It focuses on minimizing total logistic costs and greenhouse gas emissions. The research does not incorporate the real-time quality of the meat but does refer to it as an interesting future research area. Research by [10] uses a fuzzy multi-objective planner to minimize the environmental impact of a meat supply chain but again does not involve real-time meat quality into the model. A multicriteria optimization approach to optimize an RFID-based meat supply chain is used in [11]. Product quality is taken into account in the tri-criteria optimization in numbers of meat products.

Optimization of cold supply chains, focused on fresh foods in general, has received attention from the scientific community in recent years (e.g., [12], [7], [13], [14], [15], [16], [17]). Quality degradation of food in combination with minimizing costs is considered in [18]. The total cost model 
is optimized by three approaches (genetic algorithm, fuzzy genetic algorithm, and improved simulated annealing). In [19], both quality and costs are considered and a mixedinteger linear programming (MILP) model for production and distribution planning is presented. The products' change of location and change of quality are two different aspects, they can affect each other, but do not depend on each other.

The literature review shows little research is available on fresh food supply chains, especially into the fresh food supply chain of meat. This might be due to the necessity of mathematical models for fresh food quality. These models will make simultaneously modeling logistic processes while maintaining quality of the fresh food in a supply chain possible. However, these mathematical models are not widely available. Some research using MILP strategies on the optimization of the supply chain of fresh food has been carried out. The quality-aware optimization of a meat supply chain has, however, yet to receive attention from the scientific community.

\section{MODELING}

In this section, first assumptions considered in this study are listed. Next, the quality- and logistic aspects of the meat supply chain are described. Then, the quality-aware model is formalized for scheduling in the specific case of Irish lamb.

\section{A. Assumptions}

- The considered supply chain of Irish lamb is characteristic for all meat supply chains.

- Information regarding quality and temperature of meat is predictable and available.

- Quality and temperature of a unit of meat is homogeneous within a unit.

- Production facilities only produce products that satisfy the quality constraints.

- Demand from customers is known in advance.

- Shipments are made at the end of the periods, and are measured in product units. Here, we also assume that related cooling and distribution costs can be expressed per product unit.

- Parameters are stable and do not change during simulation.

- Retailers refrigerate at $5^{\circ} \mathrm{C}$.

\section{B. Cooling Costs}

Fresh meat and meat products have to be processed, stored, and transported in cold conditions within a temperature range of $+2^{\circ} \mathrm{C}$ to $+7^{\circ} \mathrm{C}$, depending on the type of meat [4]. However, this is not always the case and temperatures can range from $+2{ }^{\circ} \mathrm{C}$ to ambient temperature, for instance, when there is no place left in a refrigerator. In this study we will consider the units of meat $m$ to be euro pallets with a maximum payload of 1000 kilogram. A total number of $M$ units are present in the supply chain. These can be refrigerated between $+2{ }^{\circ} \mathrm{C}$ to an ambient temperature of +20 ${ }^{\circ} \mathrm{C}$. We therefore consider 19 levels of temperature, which will be indicated by $f \in F$. The cost of storing meat and meat products depends on the temperature. Assuming one can neglect energy losses, the coefficient of performance (COP) for refrigeration can be used (1) [20]:

$$
C O P=\frac{Q_{L}}{W}=\frac{T_{L}}{T_{H}-T_{L}}
$$

where $Q_{L}$ is the heat transferred from a lower temperature environment to a higher temperature environment, $W$ is the work which needs to be put into the cycle, $T_{H}$ is the higher temperature (environment), and $T_{L}$ is the lower temperature in the refrigerator. $T_{H}$ and $T_{L}$ are both measured in Kelvin [19]. Meat products can be stored between $275.15 \mathrm{~K}\left(2^{\circ} \mathrm{C}\right)$ and $293.15 \mathrm{~K}\left(20^{\circ} \mathrm{C}\right)$, which is the considered ambient temperature. If we now give $275.15 \mathrm{~K}$ the relative cost $\left(p_{f}\right)$ of 1 , we can calculate the relative costs of $3^{\circ} \mathrm{C}$ to $20^{\circ} \mathrm{C}$. For example, for $2{ }^{\circ} \mathrm{C}$ we receive a COP value of 16.6 from (1). When we now consider $3^{\circ} \mathrm{C}$, we receive a COP of $276.15 /(293.15-276.15)=17.7$ so the relative cost $\left(p_{f}\right)$ of $3{ }^{\circ} \mathrm{C}$ as compared to $2{ }^{\circ} \mathrm{C}$ are $15.3 / 16.2=0.94$.

\section{Quality Aspects}

The quality of meat can be represented by a first order equation. One can estimate the quality of meat at a certain moment of time in a certain location in the supply chain based on an initial quality $\left(q_{0}\right)$ [3]. In general such a first order reaction looks like (2):

$$
q=q_{0}-\exp \left(\sum_{i=1}^{p} k_{i} t_{i}\right)
$$

Every type of meat deteriorates according to different dynamics depending on size, origin, color, etc. This study will use a mathematical shelf life model describing the deterioration of Irish lamb presented in [21]. The quality of a unit of Irish lamb is indicated using the Quality Index (QI) shown in (3):

$$
Q I\left(t_{i}, T_{i}\right)=\sum_{i} \exp ^{\left(-9876.57 * \frac{1}{T_{i}}+30.5\right)} \cdot \Delta t_{i}+1,
$$

where $T_{i}$ is the temperature of refrigeration at node $i$ and $t_{i}$ is time. When the QI has a value of 4.0, the product is discarded, because this is the limit of acceptance level for this product. This study will use a discrete-time model, based on time steps of a day. Furthermore, in order to present readable values of the remaining quality, we multiply both the initial quality as well as the degradation factors by 10 . A unit of Irish lamb $m$ will thus have an initial quality of 30 and will degrade according to (4), which gives the quality deterioration per day at node $i$, depending on the temperature level $f_{i}$ as:

$$
\Delta q_{m i f}=24 \cdot \exp ^{\left(-9876.57 * \frac{1}{f_{i}}+30.5\right)}
$$

which brings the remaining quality of unit $m$ at time step $k+1$ to be equal to:

$$
q_{m}(k+1)=q_{m}(k)-\sum_{(i, j) \in E} \Delta q_{m i f}(k) \cdot u_{m i j}(k) .
$$




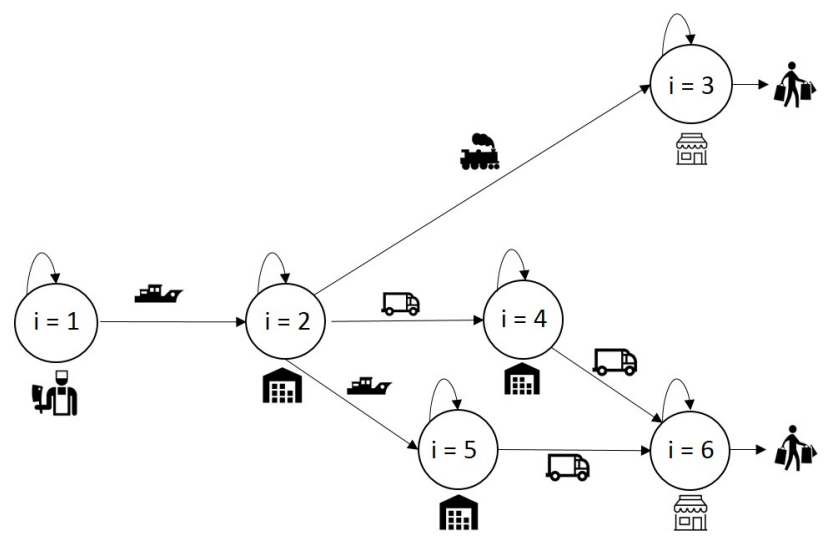

Fig. 1. Considered supply chain model with producer (1), warehouses (2, $4,5)$, and retailers $(3,6)$.

\section{Logistic Aspects}

A meat supply chain consists of consecutively: farms; abattoirs; producers; one or multiple warehouses; retailers; and finally the customers. Livestock does not have to be cooled, so farms are not considered in the cold supply chain. Furthermore, contemporary abattoirs and producers are often integrated into one facility where livestock enters and products leave to go to the next node in the supply chain. In the supply chain of Irish lamb considered in [21], the supply chain consists of consecutively: a producer, a few intermediate warehouses, and a retailer. In this study this supply chain is represented by a producer, two warehouses, and a retailer. In this paper we extend the aforementioned supply chain with more route choices and destinations (retailers), as shown in Fig. 1. This allows supply chain planners to consider transport modalities other than truck, and to make use of more choices of destinations.

In the extended supply chain, the logistic status of units of meat is represented by stages. The model provides options for different modes of transport between these nodes (stages). These transport modes are barge-, truck- and train transport, each of which has its own characteristics.

The dynamics of one meat unit in the supply chain is denoted by a directed graph $G=\{N, E\} . N$ are the nodes indicated by indices $i$, and include every location a unit of meat $m$ can be in. $E$ is the collection of arcs $(i, j)$, which stands for possible transitions between locations in the collection $N$. Notation $l_{m i}(k)=1$ is used when particular unit $m$ is at node $i$ at time step $k$ [22]. $u_{m i j}(k)=1$ is used when the decision $u$ is made to transport the unit of meat $m$ from node $i$ to node $j$ at time step $k . u_{m i i}(k)=1$ is used when the decision $u$ is made to keep unit of meat $m$ at node $i$ at time step $k$. So, $l_{m j}(k+1)=1$ represents that unit of meat $m$ will be at node $j$ at time step $k+1$. The collection $B$ contains all possible modes of transport $b$. In the collection $P$ all predecessor nodes of current node $i \in N$ are listed, in collection $S$ all successor nodes of the current node $i \in N$ are listed.

Variables related to each unit $m$ are linked by three types of constraints, namely logistics, demand from retailers, and quality constraints. Using the introduced notation, we next provide all the constraints of the model followed by their motivations:

$$
\begin{aligned}
& q_{m}(k+1)=q_{m}(k)-\sum_{(i, j) \in E} \sum_{f \in F} \Delta q_{m i f}(k) \cdot u_{m i j}(k), \\
& \forall i \in N, m \in M, k \in\{1,2, \ldots, K\} . \\
& \omega\left(1-\sum_{i \in P} u_{m i i}(k)\right)+q_{m}(k) \geq q_{i, \text { min }}, \\
& \forall i \in N, m \in M, k \in\{1,2, \ldots, K\} . \\
& \omega\left(1-\sum_{i \in N} \sum_{j \in N} u_{m i j}(k)\right)+q_{m}(k) \geq 0, \\
& \forall m \in M, k \in\{1,2, \ldots, K\} \text {. } \\
& w_{i}=\sum_{k=1}^{K} \sum_{m \in M} u_{m i j}(k), \forall i \in N, j=w . \\
& \sum_{f \in F} \Delta q_{m i f}(k)=u_{m i j}(k), \\
& \forall m \in M, i \in N, k \in\{1,2, \ldots, K\} \\
& \sum_{i \in N} l_{m i}(k)=1, \forall m \in M, k \in\{1,2, \ldots, K\} . \\
& \sum_{p \in P(i) \cup\{i\}} u_{m p i}(k)=\sum_{j \in S(i) \cup\{i\}} u_{m i j}(k+1), \\
& \forall m \in M, i \in N, k \in\{1,2, \ldots, K\} . \\
& \sum_{j \in S(i) \cup\{i\}}\left(t_{b}^{l e a d} \cdot u_{m i j}(k)\right)-a_{m j}-\omega u_{m j j}(k+1) \leq 0, \\
& \forall m \in M, i \in N, k \in\{1,2, \ldots, K\} . \\
& a_{m j}(k)=\sum_{j \in S(i) \cup\{i\}} \sum_{\tau=1}^{k} u_{m i j}(\tau), \\
& \forall m \in M, i \in N, k \in\{1,2, \ldots, K\} \text {. } \\
& s_{i}(k)=\sum_{m \in M} \sum_{j \in S(i) \cup\{i\}} u_{m i j}(k), \forall i \in R, k \in\{1,2, \ldots, K\} . \\
& d_{i}(k)=g_{i}(k)+g_{i}^{C}(k), \forall i \in R, k \in\{1,2, \ldots, K\} . \\
& s_{i}(k)=g_{i}(k), \forall i \in R, k \in\{1,2, \ldots, K\} .
\end{aligned}
$$

Constraints (6)-(10) are quality constraints. Constraint (6) tracks the quality of units of meat $m \in M$, constraint (7) makes sure that if a unit of meat $m$ is not up to the quality requirements of any of the nodes, it gets discarded. Here, $\omega$ is a large positive value. Constraint (8) will make sure that if the quality is zero, the meat will get disposed of, note this can only happen at the retailer. Constraint (9) counts the number of units of meat that have been spoiled. Finally, constraint (10) obliges the model to choose a temperature level $f$ at which a unit of meat $m$ is stored. Different temperatures will 
impact the rate of deterioration of the units of meat at that node.

Constraints (11)-(17) are logistic constraints. Constraint (11) makes sure units of meat $m$ can only appear on one place each time step. Constraint (12) makes sure that units of meat can only follow arcs $(i, j)$. Constraints (13) and (14) make sure that a unit of meat $m$ is constrained from moving from node $i$ to $j$, before having stayed at node $i$ for $t_{b}^{\text {lead }}$ time steps. Constraint (14) will count the number of time steps the meat is already at the facility, before it can actually be used by the distribution centre in order to simulate transport times.

Constraint (15) makes it possible to connect the demand and supply of nodes $i$ with decision variable $u_{m i j}$. Constraint (16) shows in which ways demand $d_{i}(k)$ can be fulfilled. The term $g_{i}$ specifies the demand can be fulfilled while the term $g_{i}^{C}$ denotes the number of units of meat within the demand that can not be fulfilled. Constraint (17) links the supply, which can be determined using (15), to the demand.

\section{E. Objective Function}

We consider an objective function over a finite time period $K=\left\{1,2, \ldots, N_{e}\right\}$ :

$$
\begin{aligned}
J & =\sum_{i \in N} \sum_{k \in K} \sum_{f \in F} \mathbf{g}_{i}(k) \cdot P_{q} \\
& -\sum_{i \in N} \sum_{k \in K} \sum_{m \in M} \sum_{f \in F} \mathbf{q}_{m i f}(k) \cdot p_{f} \Delta \\
& -\sum_{i \in N} \sum_{k \in K} \sum_{b \in B} \sum_{m \in M} \sum_{(i, j) \in E} \mathbf{u}_{m i j}(k) \cdot P_{b} \\
& -\sum_{i \in N} \sum_{k \in K} \sum_{m \in M} \sum_{j \in w} \mathbf{u}_{m i j}(k) \cdot \xi \\
- & \sum_{i \in N} \sum_{k \in K} \mathbf{g}_{i}^{C}(k) \cdot \mu \\
- & \sum_{i \in N} \sum_{k \in K} \sum_{b \in B} \sum_{m \in M} \sum_{(i, j) \in E} \mathbf{u}_{m i j}(k) \cdot E_{b}
\end{aligned}
$$

in which $P_{q}$ is the price that is received for selling a product of a certain quality $q$. This quality and thus also the price can, in certain scenarios, decrease with time depending on the temperature level chosen. The cooling costs are calculated using parameter $p_{f}$, which is the cost of cooling a unit of meat $m$ at a certain temperature level $f, P_{b}$ is the transport costs of using transport mode $b$ to transport unit of meat $m$ over $\operatorname{arc}(i, j)$. The parameters $\xi, \mu$, and $E_{b}$ are used for penalties on respectively: producing waste, unmet demand, and producing $\mathrm{CO}_{2}$ emissions using transport mode $b$ to transport unit of meat $m$ over arc $(i, j)$.

The objective function considers income for selling units of meat to retailers, cooling costs, transport costs, and penalties for waste, unmet demand and emissions. The qualityaware model thus considers both the logistic activities as well as the quality of products. The combination of the objective function and the constraints form an MILP problem, optimize the objective function (18) subject to constraints (6)-(17). The used decision variables are $\mathbf{u}_{m i j}, \mathbf{g}_{i}, \mathbf{g}_{i}^{C}, \Delta \mathbf{q}_{m i f}$ for all $k \in\left\{1,2, \ldots, N_{e}\right\}$.

\section{SIMULATION EXPERIMENTS}

As mentioned there currently are a lot of problems in the contemporary supply chain, which result in waste of meat. This waste of meat is the primary problem this model is designed to tackle. The deterioration of meat is highly influenced by the temperatures at which the meat is being stored and distributed, as shown in Section III-C. The quality-aware controller will accurately keep track of the quality of every unit of meat using the presented mathematical model. In this section, the contemporary control will be compared with the quality-aware controller in two different scenarios. The first scenario considers the normal operation procedure of a contemporary supply chain of Irish lamb as sketched in [21]. In this study, this supply chain will be represented by 4 nodes, namely the producer, two warehouses, and a retailer. The second scenario considers the proposed extended supply chain shown in Fig. 1. First, the contemporary control will be used to simulate both scenarios. Next, the qualityaware controller using an MILP strategy will be used to show the potential of varying the cooling temperature in both scenarios. Parameters used in the simulations are shown in Table I, note that units of meat have a different initial quality. In the second scenario the demand of the retailer, $d_{r}(k)$, will change from the value shown in Table I to one unit of meat at day $7,8,9$, and 10 for both retailers. The objective function (18) is used to obtain a value that indicates the profit and thus performance of both control procedures. The total number of decision variables in these simulations is approximately 10000. The experiments are carried out using Matlab R2017a, on a laptop with Intel Core i7, 8GB RAM, and Windows 7-64bit. The optimization problems are solved using the intlinprog solver integrated in Matlab R2017a.

\section{A. Contemporary scheduling}

In contemporary supply chains, high variations in temperature occur in the contemporary supply chain, however, the exact temperature history is not available. We assume the scheduler sets the cooling temperature at $+7^{\circ} \mathrm{C}$. Because this is the maximum temperature allowed by regulations. This amounts to a total cooling cost of $€ 594.74$ in both scenarios.

TABLE I

SIMULATION PARAMETERS

\begin{tabular}{|l|l|}
\hline Parameter & Value \\
\hline$M$ & 8 \\
\hline$N$ & 6 \\
\hline$N_{e}$ & 10 \\
\hline$b$ & {$[1,2,3]=[$ Barge, Train, Truck] } \\
\hline$t_{b}^{\text {lead }}$ & {$[2,1,1][$ days $]$} \\
\hline$P_{b}$ & {$[370,1340,1870][€]$} \\
\hline$E_{b}$ & {$[3.60,2.55,7.20][€]$} \\
\hline$P_{q}$ & $10000[€]$ \\
\hline$\mu$ & $500[€]$ \\
\hline$\xi$ & $500[€]$ \\
\hline$p_{f}$ & $9.60[€]$ \\
\hline$d_{r}(k)$ & $d_{r}(7)=2, d_{r}(8)=2, d_{r}(9)=2, d_{r}(10)=2$ \\
\hline$f$ & {$[2, \ldots, 20]{ }^{\circ} \mathrm{C}$} \\
\hline$q_{m, \text { initial }}$ & {$[30,30,28,28,26,26,24,24]$} \\
\hline$q_{r, \text { min }}$ & $16 \forall r$ \\
\hline
\end{tabular}


Scenario 1: The current scheduler is not able to inspect the remaining quality of the units of meat, so it will just try to obey to the demand. This results in one unit of meat being spoiled before arriving at the retailer. The highest possible profit for this scenario is $€ 35,438.86$.

Scenario 2: In this scenario, the scheduler is able to choose between different types of transport modes, shown in Fig. 1, which could increase profit. The scheduler is, however, still not able to inspect the quality. The locations of every unit of meat are shown in Fig. 2, where stages 3 and 6 represent retailers. The controller delivers unit of meat 8 at time step 10, when this unit of meat is already spoiled, which causes penalties for unmet demand as well as waste to be incurred. The highest possible profit for this scenario is $€ 51,057.46$.

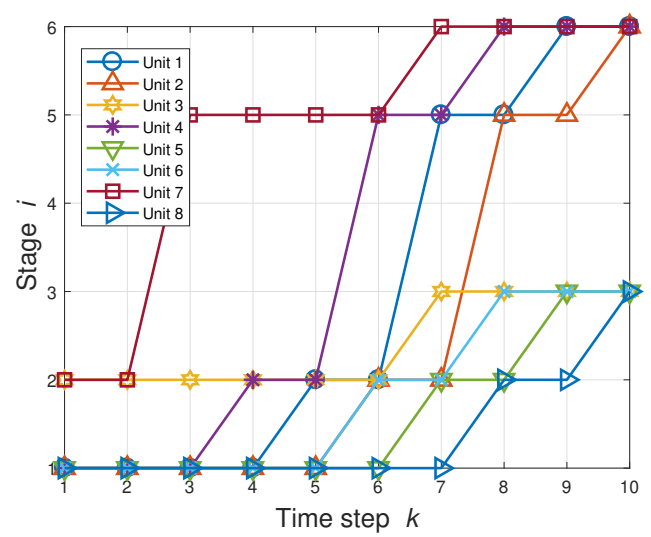

Fig. 2. Location of units of meat at every time step for the supply chain scheduling sketched in [21].

\section{B. Quality-Aware Scheduling}

The optimal controller can decide at which temperatures the meat will be cooled. The controller is also aware of the quality of units of meat and thus can accurately predict the remaining shelf life when the meat is delivered to a retailer. An extra constraint is introduced for this controller, retailers will require a remaining shelf life of at least 10 days [14].

Scenario 1: The quality aware controller causes the waste to be eliminated in this scenario which increases the profit to $€ 46,269.28$. The total cooling costs are $€ 768.32,32 \%$ higher as compared to the contemporary control, but the total profit increases with $30 \%$.

Scenario 2: In this scenario, we again consider Fig. 1, in which the scheduler can choose different transport modes. The locations of all units of meat are shown in Fig. 3, where nodes 3 and 6 again represent retailers. Observe the controller now delivers the units of meat with the lowest quality ( 7 and 8) first. Cooling levels of every unit of meat at every time step are shown in Fig. 4. Note that in the simulation, when a unit of meat reaches the retailers, the unit is considered delivered. This causes the outliers in time steps 8, 9, and 10 in Fig. 4. Meat delivered at time step 7 can choose any level of cooling without deteriorating. The remaining quality of every unit of meat is shown in Fig. 5, a remaining quality, $q$, of 16 corresponds to a shelf life of 10 days. The profit of this scenario is $€ 61,887.88$. The total cooling costs are $€ 764.32$, an increase of $28 \%$, while the total profit increases with $21 \%$ as compared to the contemporary control.

This means an increase of profit of up to $30 \%$ can be reached when the controller can decide over temperature levels, as compared to the contemporary supply chain control sketched in [21]. In a meat supply chain, efficiency can be improved by considering quality information in the optimization of logistic processes by controlling the cooling temperatures where meat is stored. The controller used in this simulation does, however, assume perfect knowledge regarding both quality as well as logistic aspects.

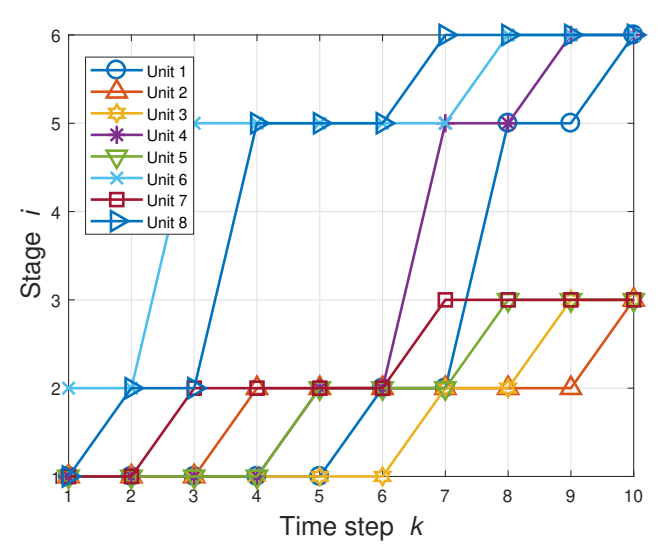

Fig. 3. Location of units of meat at every time step. The different nodes are shown on the y-axis.

\section{CONCLUSIONS AND FUTURE RESEARCH}

Although information about temperature can be made available in real-time by ever-improving sensors and communication technologies, waste of meat throughout supply chains is still high. Especially when one considers the amount of resources needed to produce one kilogram of meat, the necessity for improving this particular supply chain becomes more evident. This paper presents a quality-aware modeling method for meat supply chain operations. The method considers both the quality and logistic aspects of the

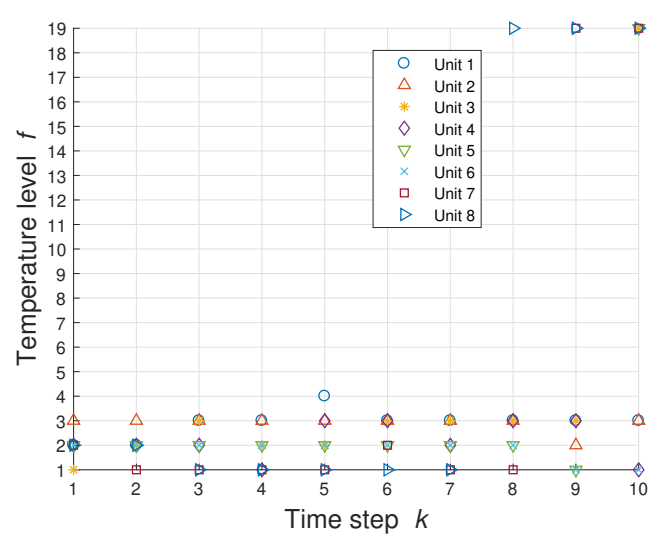

Fig. 4. Cooling levels of every unit of meat at every time step. 

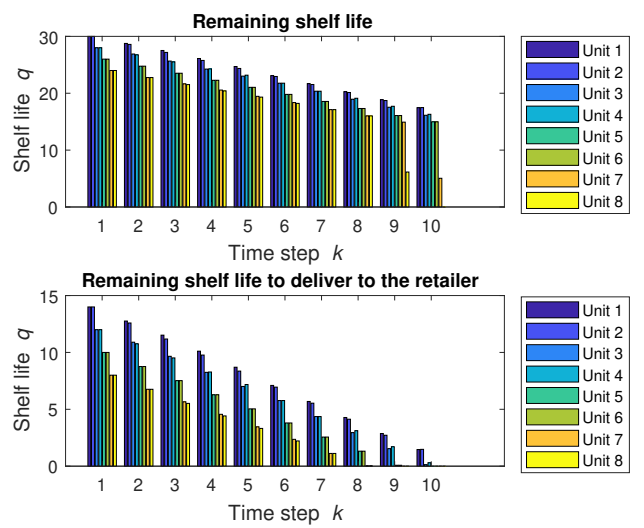

Fig. 5. The top figure shows the total remaining shelf life of every unit of meat. The bottom figure shows the remaining shelf life for the meat to be accepted by the retailer.

goods being transported. Especially, a temperature control feature is proposed with the modeling method, allowing controllers to adjust the cooling temperature for meat products. A contemporary control strategy and an quality-aware control strategy are used in numerical demonstrations to determine supply chain operations. The results illustrate that a sustainable approach towards optimization of supply chains does not necessarily imply costs. Moreover, the quality-aware controller shows it is indeed possible to decrease, or even eliminate waste in the supply chain of meat. The proposed method provides potential for controllers to improve meat supply chain operations by considering both quality and logistic features of the goods, with the knowledge of the system.

We also find out that in current supply chains, cooling costs do not have a large impact on the decisions made by supply chain planners, because of their low cooling costs. However, with the ever growing concern of $\mathrm{CO}_{2}$ emission, the drawback of refrigerating cannot be neglected anymore. Once carbon tax is imposed, reducing refrigeration can be an important asset. Further extensions of this paper should consider multi-objective optimization, different types of meat, and heterogeneous quality in a unit. Further research also includes adopting other real-time control strategies such as model predictive control in order to react to disturbances in the supply chain. Stochastic and fuzzy formulations of the problem could also be explored to deal with uncertainty. Yet another interesting topic which can also be considered in future research is that of distributed control strategies. A limitation of this work is that it is assumed to have perfect knowledge on the system parameters, which is shared among all the involved stakeholders, something unlikely in a real setting.

\section{REFERENCES}

[1] A. Stenmarck, C. Jensen, T. Quested, G. Moates, M. Buksti, B. Cseh, S. Juul, A. Parry, A. Politano, B. Redlingshofer, and others, Estimates of European food waste levels. IVL Swedish Environmental Research Institute, 2016.

[2] H. Ritche and M. Roser, "Meat and Seafood Production \& Consumption," Our World in Data, 2017.
[3] T. P. Labuza and others, Shelf Life Dating of Foods. Food \& Nutrition Press, Inc., 1982.

[4] V. Raab, B. Petersen, and J. Kreyenschmidt, "Temperature monitoring in meat supply chains," British Food Journal, vol. 113, no. 10, pp. 1267-1289, 2011.

[5] S. Arason, E. I. Ásgeirsson, B. Margeirsson, S. Margeirsson, P. Olsen, and H. Stefánsson, "Decision support systems for the food industry," in Handbook on Decision Making, pp. 295-315, Springer, 2010.

[6] M. H. Zwietering, I. Jongenburger, F. M. Rombouts, and K. Van't Riet, "Modeling of the bacterial growth curve," Applied and Environmental Microbiology, vol. 56, no. 6, pp. 1875-1881, 1990.

[7] S. Mercier, S. Villeneuve, M. Mondor, and I. Uysal, "Timetemperature management along the food cold chain: A review of recent developments," Comprehensive Reviews in Food Science and Food Safety, vol. 16, no. 4, pp. 647-667, 2017.

[8] M. L. Tamplin, "Integrating predictive models and sensors to manage food stability in supply chains," Food Microbiology, vol. 75, no. 1, pp. 90-94, 2018.

[9] M. Soysal, J. M. Bloemhof-Ruwaard, and J. der Vorst, "Modelling food logistics networks with emission considerations: The case of an international beef supply chain," International Journal of Production Economics, vol. 152, pp. 57-70, 2014.

[10] A. Mohammed and Q. Wang, "The fuzzy multi-objective distribution planner for a green meat supply chain," International Journal of Production Economics, vol. 184, pp. 47-58, 2017.

[11] A. Mohammed and Q. Wang, "Multi-criteria optimization for a costeffective design of an RFID-based meat supply chain," British Food Journal, vol. 119, no. 3, pp. 676-689, 2017.

[12] X. Lin, R. R. Negenborn, and G. Lodewijks, "Survey on operational perishables quality control and logistics," in Proceedings of the 6th International Conference on Computational Logistics, (Delft, The Netherlands), pp. 398-421, Springer, 2015.

[13] F. Dabbene, P. Gay, and N. Sacco, "Optimisation of fresh-food supply chains in uncertain environments, Part I: Background and methodology," Biosystems Engineering, vol. 99, no. 3, pp. 348-359, 2008.

[14] X. Wang and D. Li, "A dynamic product quality evaluation based pricing model for perishable food supply chains," Omega, vol. 40, no. 6, pp. 906-917, 2012.

[15] X. Lin, R. R. Negenborn, and G. Lodewijks, "Predictive quality-aware control for scheduling of potato starch production," Computers and Electronics in Agriculture, vol. 150, pp. 266-278, 2018.

[16] M. Bortolini, M. Faccio, E. Ferrari, M. Gamberi, and F. Pilati, "Fresh food sustainable distribution: cost, delivery time and carbon footprint three-objective optimization," Journal of Food Engineering, vol. 174, pp. 56-67, 2016.

[17] R. Zhao, Y. Liu, N. Zhang, and T. Huang, "An optimization model for green supply chain management by using a big data analytic approach," Journal of Cleaner Production, vol. 142, pp. 1085-1097, 2017.

[18] D. Nakandala, H. Lau, and J. Zhang, "Cost-optimization modelling for fresh food quality and transportation," Industrial Management \& Data Systems, vol. 116, no. 3, pp. 564-583, 2016.

[19] A. Rong, R. Akkerman, and M. Grunow, "An optimization approach for managing fresh food quality throughout the supply chain," International Journal of Production Economics, vol. 131, no. 1, pp. 421-429, 2011.

[20] S. K. Wang and S. K. Wang, Handbook of Air Conditioning and Refrigeration, vol. 49. McGraw-Hill New York, 2000.

[21] M. Mack, P. Dittmer, M. Veigt, M. Kus, U. Nehmiz, and J. Kreyenschmidt, "Quality tracing in meat supply chains," Philosophical Transactions Royal Society A, vol. 372, no. 2017, p. 20130308, 2014.

[22] X. Lin, R. R. Negenborn, M. B. Duinkerken, and G. Lodewijks, "Reducing Unmet Demand and Spoilage in Cut Rose Logistics: Modeling and Control of Fast Moving Perishable Goods," Transportation Research Record, 2018. 